\title{
Tumor-associated Collagen Signatures: An Insight
}

\author{
${ }^{1}$ V Pavithra, ${ }^{2}$ SV Sowmya, ${ }^{3}$ Roopa S Rao, ${ }^{4}$ Shankargouda Patil, ${ }^{5}$ Dominic Augustine \\ ${ }^{6}$ Vanishri C Haragannavar, ${ }^{7}$ Shwetha Nambiar
}

\begin{abstract}
Tumor microenvironment has a diverse capability to induce both beneficial and adverse consequences for tumorigenesis. It is a multifactorial process induced by the imbalance in the tumor cells and extracellular matrix (ECM). Collagen, the main component of ECM, is traditionally regarded as a passive barrier to resist tumor cell invasion. In recent years, collagen is marked to have its pivotal role to initiate and promote tumor progression. Remodeling of collagen has been appreciated in various benign and malignant tumors. These alterations can be identified and demonstrated as tumor-associated collagen signatures that can be demonstrated using second harmonic generation imaging. Recognition of these characteristic changes in the organization of collagen fiber may potentially serve as an early diagnostic marker in various pathological processes, such as hyperplastic, dysplastic, and cancerous tissues. This review focuses on the physiological and pathological orientation of collagen fibers in relation to epithelium that acts as an image-based biomarker.
\end{abstract}

Keywords: Collagen reorientation, Second harmonic generation microscope, Tumor microenvironment.

How to cite this article: Pavithra V, Sowmya SV, Rao RS, Patil S, Augustine D, Haragannavar VC, Nambiar S. Tumorassociated Collagen Signatures: An Insight. World J Dent 2017;8(3):224-230.

Source of support: Nil

Conflict of interest: None

\section{INTRODUCTION}

Tumor microenvironment plays a key role in multiple stages of disease progression of cancer and has enthused researchers in recent years. ${ }^{1}$ The mechanism of collagen remodeling in the tumor microenvironment is an area, i.e., minimally explored. ${ }^{2}$ The importance of understanding the significance of collagen in tumor stroma is enhanced with the advent of novel markers, such as tumor-associated collagen signature (TACS) that helps in characterizing tumors. ${ }^{3}$

\footnotetext{
1-3,5-7 Department of Oral Pathology and Microbiology, Faculty of Dental Sciences, M. S. Ramaiah University of Applied Sciences Bengaluru, Karnataka, India

${ }^{4}$ Division of Oral Pathology, Department of Maxillofacial Surgery and Diagnostic Sciences, College of Dentistry, Jazan University Jazan, Kingdom of Saudi Arabia

Corresponding Author: $\vee$ Pavithra, Department of Oral Pathology and Microbiology, Faculty of Dental Sciences, M. S. Ramaiah University of Applied Sciences, Bengaluru, Karnataka India, Phone: +917406027039, e-mail: pavithra9388@gmail.com
}

Desmoplastic stroma has already been proved to play a role in contractility of tumor cells inducing tensiondependent extracellular matrix (ECM) alterations resulting in the reorientation of collagen fibrils. ${ }^{4}$ Factors, such as cytokines, growth factors, integrin signaling, and matrix metalloproteinases (MMPs), influence tumor invasion and metastasis. ${ }^{5}$ However, the precise role of collagen as a part of microenvironment in tumor progression needs to be investigated. ${ }^{6}$

Normally, collagen is demonstrated by routine hematoxylin and eosin (H\&E) stain and special stains, such as Masson's Trichrome, Mallory's, Picrosirius red staining, and immunohistochemistry (COL-1, COL-3). ${ }^{7}$ However, to analyze TACS, second harmonic generation (SHG) microscopy is preferred as a quantitative modality for imaging collagen in a diverse range of tissue diseases, such as cancer, fibrosis, and connective tissue disorders, as SHG signal is highly sensitive to collagen-associated changes. ${ }^{8}$

\section{COLLAGEN STRUCTURE}

Rich and Crick $^{9}$ in 1955 proposed collagen to be the main structural protein forming a characteristic triple helix of three polypeptide chains. Members of the collagen family form these supramolecular structures in the ECM, although their size, function, and tissue distribution vary considerably. ${ }^{10}$

Each polypeptide chain of triple helix comprises $\alpha$-chain twisted into a left-handed helix. The polypeptide chain consists of 3.3 amino acid residues per turn, and each turn is separated by $2.9 \AA$. Three $\alpha$-chains are wound into a right-handed super helix, forming a single collagen molecule of $300 \mathrm{~nm}$ in length and $1.4 \mathrm{~nm}$ in diameter. ${ }^{10}$

\section{Amino Acid Sequence}

A single $\alpha$-chain consists of glycine at every third position as glycine is the smallest residue which accommodates in the limited spaces available in the central core. This repeating sequence is denoted as (Gly-X-Y)n, essential for the establishment of the triple helix. ${ }^{10}$

\section{Quarter-staggered Array}

The tropocollagen molecules are arranged in a quarterstaggered array. These molecules are aligned in a row by a distance of $400 \AA$ and adjacent row by $680 \AA$ in a banding pattern forming repeated sequences of overlap 
and gap regions. Cross sections of molecules comprise to form the overlap region, whereas gap region contains only four molecules. Once the collagen molecule is assembled into fibrils, there is a shift in the molecule by a distance $D$ $(65-67 \mathrm{~nm})$ corresponding to the adjacent tropocollagen with the overlap region being $0.4 \mathrm{D}$ and the gap region being $0.6 \mathrm{D} .^{11}$ This being noncentrosymmetric structure of collagen may be effective in generating the second harmonics well appreciated for the past 20 years. Although collagen-specific dyes stain various types of collagen, SHG has the potential to differentiate by stronger secondharmonic signals in type I than type III collagen. ${ }^{8}$

\section{Biosynthesis of Collagen}

The biosynthetic pathway responsible for collagen production is complex as specific gene is responsible for each type of collagen. ${ }^{12}$ Collagen biosynthesis is represented in Figure 1.

\section{COLLAGEN REMODELING IN TUMOR MICROENVIRONMENT}

The tumor microenvironment undergoes persistent architectural and molecular changes, such as degradation, increased deposition, and stiffening. Furthermore, immune infiltration at the tumor invasive front corresponds with collagen remodeling. ${ }^{13}$

\section{Collagen Degradation}

Collagen in normal tissue acts a physical barrier for tumor cell to invade the ECM. Collagen degradation is an essential process for tumor invasion which is mediated by MMPs. The MMPs have a direct effect on tumor growth, invasion, and angiogenesis resulting in collagen degradation responsible for tumor cells to escape and invade the ECM and further create interstitial tension to induce epithelial-mesenchymal transition (EMT). ${ }^{14}$

\section{Increased Deposition and Cross-linking of Collagen}

As the tumor progresses, there is extensive structural change in the ECM that includes increased deposition of collagen I, III, IV, fibronectin, and proteoglycans along with increased cross-linking of collagen molecules. ${ }^{15}$ Enhanced alterations in the ECM generate a restructured microenvironment to stimulate tumor progression by destabilizing cell adhesion and polarity. Simultaneously, there is enhanced growth factor signaling. This persistent ECM remodeling is characterized by linearization of interstitial collagen at the tumor invasion front that represents typical morphological change at the early stage of cancer. These changes have significant impact on tumor biology. ${ }^{15,16}$ A study conducted by Kumari et $\mathrm{al}^{17}$ demonstrated the remodeling of collagen from type I which is well differentiated to type III in poorly differentiated oral squamous cell carcinoma using Picrosirius red stain and immunohistochemistry. They concluded that remodeling significantly affects the biological behavior of the tumor by making it more aggressive in nature.

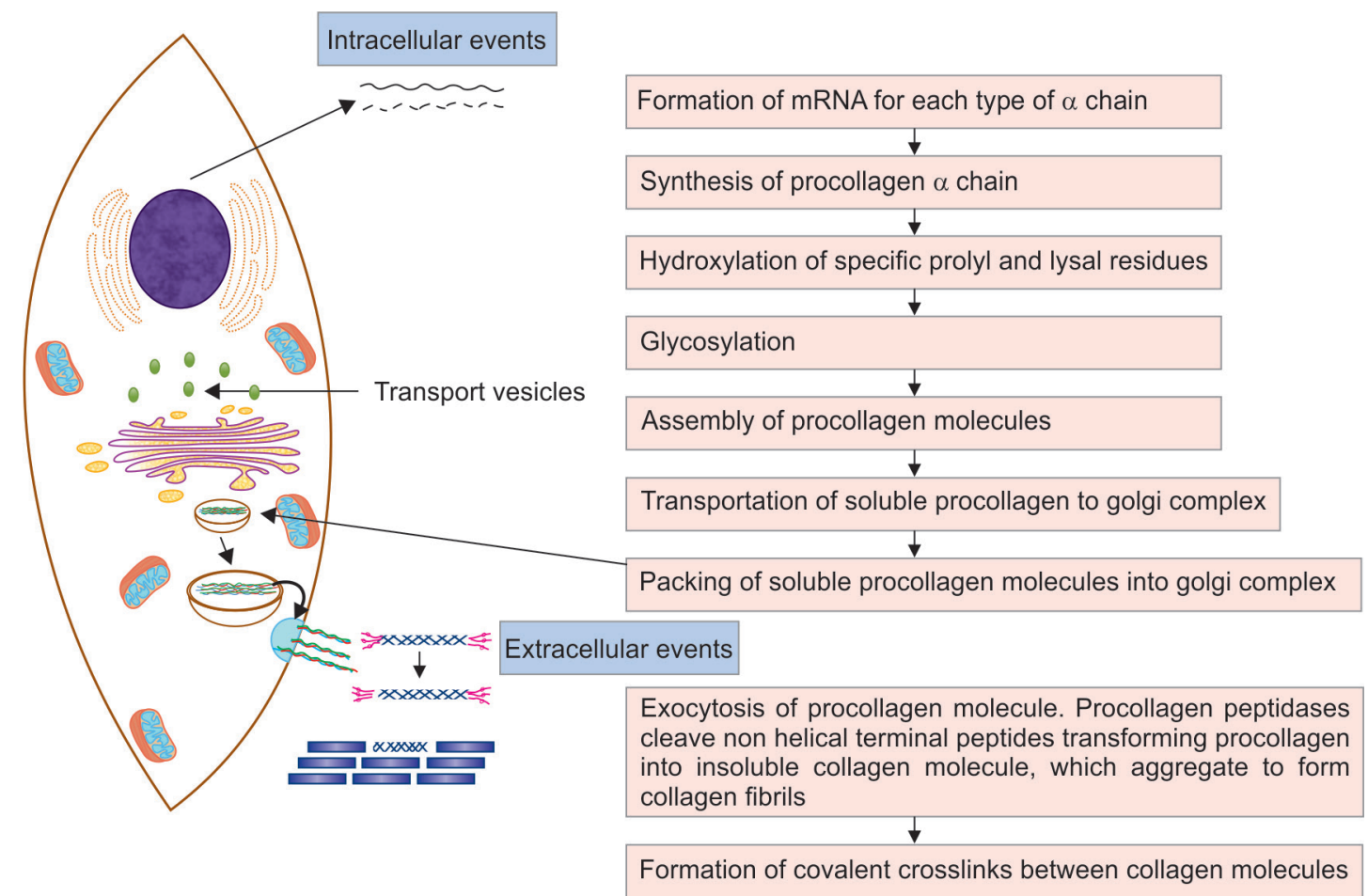

Fig. 1: Schematic representation of collagen biosynthesis ${ }^{12}$ 


\section{Protease-dependent Collagen Cross-linking based on Lysyl Oxidase}

In the tumor microenvironment, the hypoxic cells secrete lysyl oxidase that has the ability to cross-link collagens and elastin. Hence, there is enhanced insoluble collagen production and stiffening of tissue-activating integrins, thereby improving tumor cell adhesion and migration. ${ }^{18}$

\section{Protease-independent ECM Stiffening}

Other factors that influence stiffening of the matrix are glycosylation, transglutamination, increased biglycan, and proteoglycan fibromodulin deposition. ${ }^{19}$

Few models of protease-independent ECM stiffening are as follows:

- Excessive deposition of proteoglycans that induce stromal fibrosis in wounded tissues ${ }^{19}$

- Fibronectin-mediated collagen remodeling ${ }^{20}$

- Role of secreted protein acidic and rich in cysteine - a multifunctional glycoprotein, synthesized by stromal and cancer cells, has the ability to bind to collagen types I and IV inducing tumor progression. ${ }^{21}$

\section{Tension regulates MMPs Function}

As tumor expands, there is a continuous remodeling and reorientation of the collagen with subsequent increase in mechanical tension and collagen density. Degradation process of collagen in normal tissue is carried out by MMPs. However, MMPs in tumor are self-modulated. ${ }^{22}$

Initially, increased tension makes the collagen to stretch, thus making ECM protective. As the tumor proliferates extensively, tumor cells exert a traction force on collagen causing deformity in the structure. As tumor expansion reaches a critical point, tumor cells sense the gestures of massive tension resulting in the separation of collagen triple helix. ${ }^{22}$

\section{Epithelial-Mesenchymal Transition}

Epithelial-mesenchymal transition is recognized by interactions between tumor cells and ECM by primary forces that trigger the process. The triggering factor that initiates EMT is an imbalanced mechanical force at the epithelial and stromal interface that eventually leads to tumor invasion. Tumor cells adapt to the altered tension and correspondingly change the behavior of tumor microenvironment. Increase in size of the tumor causes realignment and stretching of the collagen perpendicular to the tumor boundary. ${ }^{23}$

\section{Tumor-associated Collagen Signature}

Provenzano et $\mathrm{al}^{3,24}$ first coined the term "TACS" using SHG microscope while studying murine models to assess the role of collagen alignment in tumor progression. They concluded that the mechanisms behind local invasion included matrix reorganization through guanosine triphosphatase-mediated tumor cell contractility, leading to an aligned matrix facilitating the local invasion.

Collagen can be seen wrapping individual cells in multiple directions, suggesting a role in anchoring. Literature search indicates the important interaction between the stroma and the epithelial cell, suggesting the role of fibers to apply physical restraint and mechanical signals across a thin basement membrane. While the basement membrane possesses spanning cell processes and therefore, may not completely isolate the epithelial cell, it results in microregions of direct collagen-epithelial cell interactions or combination of both scenarios. ${ }^{24}$

Tumor-associated collagen signature in the tumor microenvironment is analyzed in relation to the tumor boundary or to the surface epithelium, i.e., readily recognized in epithelial neoplasms. ${ }^{24}$

Tumor-associated collagen signature can be analyzed on routine H\&E-stained sections or intact fresh tissue specimens of patients employing SHG imaging and has the ability to distinguish normal from diseased tissue using both cellular and collagen features of the tumor microenvironment. There are three types of TACS that can be appreciated in various stages of tumorigenesis. ${ }^{24}$

\section{TACS-1}

It is characterized by dense wavy collagen bundles that serve as reliable hallmark for locating the tumor in early stages and is indicated by increased SHG signal intensity at a region near the tumor. ${ }^{24}$ Increased density of ECM is indicative of a reactive stroma that supports tumor growth. ${ }^{25}$ This type of arrangement is more appreciated in benign fibrous lesions, such as fibroma, ossifying fibromas, giant cell fibroma, and salivary gland neoplasms.

\section{TACS-2}

As the size of the tumor increases, collagen fibers surrounding the tumor tend to stretch induced by tensile stress causing linearization of the collagen bundles. These changes are represented as TACS-2 or "taut" collagen fibers. ${ }^{24}$ These types of collagen fibers are appreciated around a relatively smooth tumor boundary as seen in benign tumors.

\section{TACS-3}

Realignment of collagen bundles occurs where the collagen fibers are arranged perpendicularly from tumor border or from the surface epithelium, providing a pavement for the malignant cells to move along the fibers 
toward the stroma. Malignancies, such as squamous cell and basal cell carcinomas, show morphogenesis and contractility of the malignant cells leading to fiber realignment at the tumor boundary leading and local invasion. ${ }^{24}$

To depict the orientation of collagen fibers in TACS-1, 2 , and 3, the following stains were employed on the archival specimens retrieved from the Department of
Oral Pathology, Faculty of Dental Sciences, M. S. Ramaiah University of Applied Sciences: H\&E, Masson Trichrome, and Picrosirius red (Fig. 2).

The possible mechanisms that can be related to collagen orientation are as follows:

- An existing tumor-dense region that serves to stimulate tumor formation

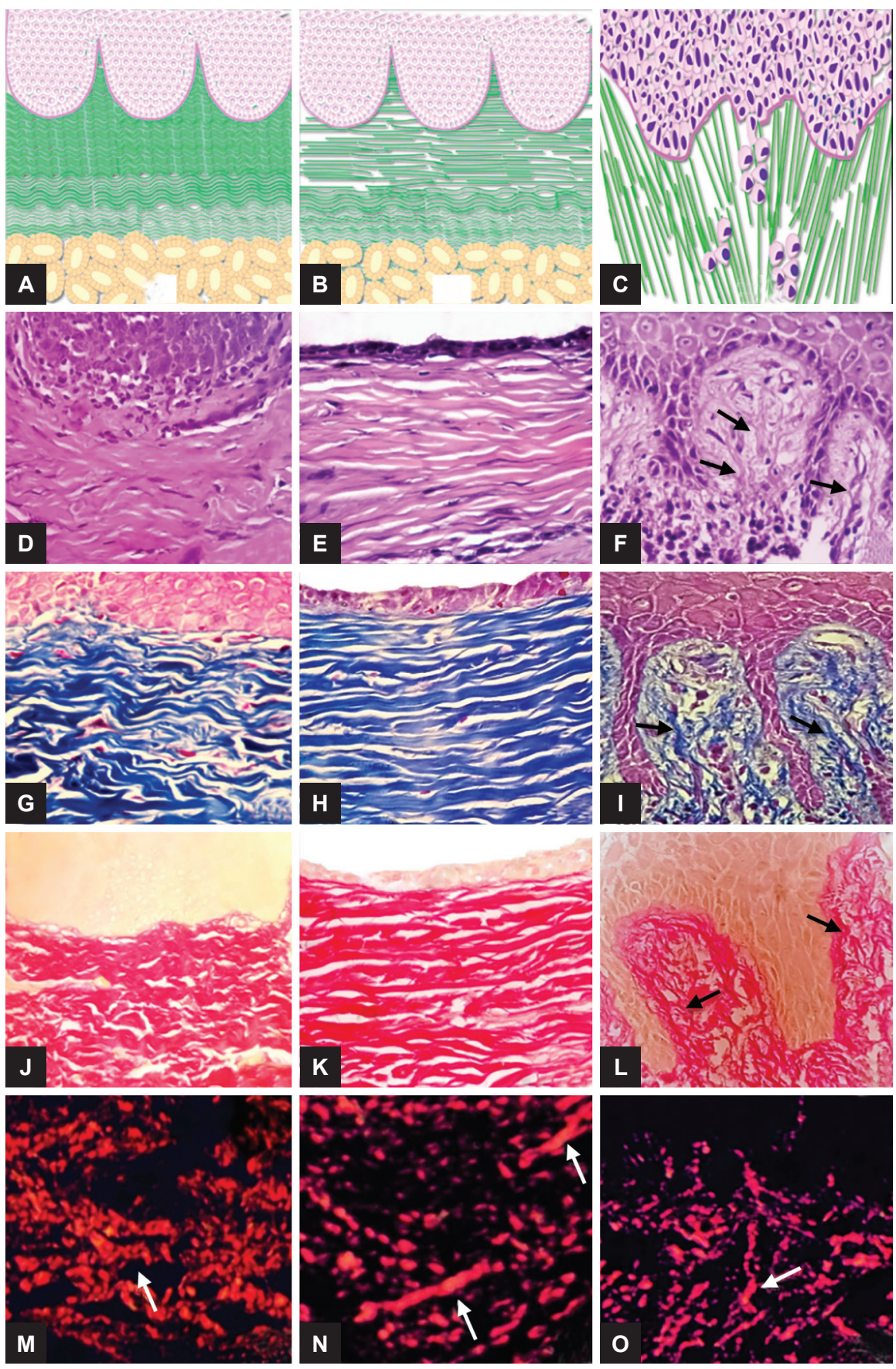

Figs 2A to O: Schematic representation of TACS: (A) TACS-1; (B) TACS-2; (C) TACS-3 ( $\times 400$ ); photomicrographs of H\&E-stained sections representative of; (D) TACS-1; (E) TACS-2; (F) TACS-3 $(\times 400)$; photomicrographs of Masson Trichrome-stained sections depicting; (G) TACS-1; (H) TACS-2; (I) TACS-3 $(\times 400)$; photomicrograph of Picrosirius red staining for collagen demonstrating; (J) TACS-1; (K) TACS-2; (L) TACS-3 under compound light microscope $(\times 400)$; photomicrograph of Picrosirius red staining for collagen demonstrating; (M) TACS-1; (N) TACS-2; and (O) TACS-3 under polarizing microscope $(\times 400)$. Collagen orientation: TACS-1 - Dense wavy collagen bundles, TACS-2 - Straight collagen bundles, TACS-3 - Collagen perpendicular to the tumor boundary or surface epithelium 
- Increased contraction of an epithelial tumor mass or motile cells at the tumor

- Boundary that triggers the dense collagen into a cluster

- Increased local collagen deposition due to activation of fibroblasts ${ }^{24}$

Various studies conducted would suffice the orientation of collagen fibers, Provanzano et al ${ }^{24}$ Conklin et al, ${ }^{25}$ and Bredfeldt et $\mathrm{al}^{26}$ quantified TACS in breast carcinoma tissues and found well-aligned TACS-positive fibers corresponding to the tumor boundary. They concluded that TACS could be used for assessing the orientation of collagen in relation to tumor stromal boundaries. In addition, they inferred TACS screening to be a potential clinical diagnostic tool, thereby TACS-3 collagen could indicate poor survival rate suggesting that quantifying collagen alignment could be an independent prognostic marker.

Brabrand $\mathrm{et}^{27}$ analyzed TACS in ductal carcinomas of varying patterns of collagen fibers wherein intratumoral region showed TACS-3, extratumoral region showed TACS-1, and juxtatumoral showed TACS-2 in relation to the tumor boundary. They inferred that TACS-3 could facilitate epithelial cell migration and tumor invasion.

Literature review recommends the ideal usage of SHG imaging to study three-dimensional structure of collagen. $^{28}$

\section{SECOND HARMONIC GENERATION IMAGING}

Freund was the first researcher in 1986 to use SHG imaging to assess the collagen in biological tissues using a rat tail tendon. Later, Miller ${ }^{12}$ and Campagnola ${ }^{28}$ in 2011 proposed the practical applications of tissue imaging and laser scanning. Subsequently, SHG microscopy has been used increasingly for imaging collagen.

\section{Principle of SHG Microscope}

The noncentrosymmetric collagen fibers interact with two photons of the incident light such that resultant photons are half the wavelength of the incident photons. The nonlinear coherent process is nonfluorescent and will specifically image collagen, where the backscattered SHG signal is easily separated from any fluorescence that may occur by the use of narrow band-pass filters centered at one-half of the laser wavelength. ${ }^{28}$

\section{Preference of SHG over Compound Microscope}

Second harmonic generation microscopy is capable of securing three-dimensional image of the tissues of several hundred microns and can obtain better data than histologic sections. Advantages of SHG over compound light microscope are acquiring nondestructive tissue images, three-dimensional imaging with additional time component $(x, y, z$, and time), obtaining images in intact, nonfixed, and nonstained tissues. ${ }^{21}$ Second harmonic generation facilitates label-free imaging and has the potential to augment current excisional biopsy protocols for melanoma diagnosis and treatment. ${ }^{28}$

Strupler et $\mathrm{al}^{29}$ and Drifka et $\mathrm{al}^{30}$ conducted studies comparing the efficiency of SHG to assess collagen in transgenic animal models with routine $\mathrm{H}$ and $\mathrm{E}$ stain, Picrosirius red, and immunohistochemical stain (COL-1) and reported that SHG microscope to be highly specific to analyze fibrillar collagens.

Matteini et $\mathrm{al}^{31}$ demonstrated thermal transitions of fibrillar collagen in the corneal stroma using SHG and polarized microscopy. Their study suggests that SHG may help in quantitative examination of biological processes and guide in postsurgical functional imaging of collagenrich tissues subjected to thermal treatments.

Thrasivoulou et al ${ }^{32}$ analyzed the unstained human melanoma tissue sections, ex vivo normal human and rat skin whole thickness biopsy tissues using SHG microscope to delineate the borders of skin cancers. Second harmonic generation imaging demonstrated collagen architecture that could be used for diagnosis and accurate demarcation of melanoma borders, thereby potentially reducing the mortality rates.

Furthermore, SHG imaging was used to study ex vivo mouse ovarian tissues of four different types: Normal, benign abnormality, dysplasia, and carcinoma by Watson et al. ${ }^{33}$ The cancerous and noncancerous tissues could be differentiated with $81.2 \%$ sensitivity and $77.8 \%$ sensitivity respectively. Hence, they recommended the usage of SHG to visualize collagen structure for early cancer detection.

Pirhonen et $\mathrm{al}^{34}$ in their study compared the ability of SHG and coherent anti-Stokes Raman scattering (CARS) microscopy to detect fibrillar collagen in advanced liver disease, such as nonalcoholic fatty liver disease. They reported that SHG imaging showed a positive correlation with different stages of liver fibrosis than CARS. Furthermore, their study demonstrated that SHG imaging detects collagen more sensitively than routine histological staging.

The above studies support the capability of collagen in generating second-harmonic signals due to its noncentrosymmetric and high crystalline structure. Furthermore, SHG microscopy of such tissues will be able to provide information about orientation, crystallinity, and morphology. Therefore, SHG-based collagen assessment may provide useful information about the role of collagen in the tumor microenvironment, mechanism of tumor progression, and metastasis to differentiate hyperplastic, dysplastic, and cancerous tissues, grading and staging of tumors. ${ }^{28}$ 


\section{CONCLUSION}

Tumor-associated collagen signature can be correlated with disease condition and is assessed by evaluating the stromal collagen with cells at the tumor-stromal interface. Identifying and characterizing TACS through observation of increased signal intensities using a nonlinear SHG microscope provides three-dimensional structure of collagen. Analysis of TACS using SHG facilitates detection of initiation and progression of tumors. Second harmonic generation is also used to study a wide spectrum of diseases, such as breast, lung, ovarian, and skin cancers by providing quantitative features about diseases related to collagen changes. Although application of TACS in clinical setting is still not in use, it may find scope in revealing important biological information about ECM alterations that accompany cancer progression and metastasis. Hence, TACS analysis may be a potential diagnostic and prognostic tool in navigating the surgeons in decision-making for surgical management of fibro-epithelial pathologies. Awareness of tumor microenvironment would provide a hope for therapeutic targets.

\section{REFERENCES}

1. Kessenbrock K, Plaks V, Werb Z. Matrix metalloproteinases: regulators of the tumor microenvironment. Cell 2010 Apr;141(1):52-67.

2. Li H, Fan $X$, Houghton J. Tumor microenvironment: the role of the tumor stroma in cancer. J Cell Biochem 2007 Jul;101(4): 805-815.

3. Provenzano PP, Inman DR, Eliceiri KW, Knittel JG, Yan L, Rueden CT, White JG, Keely PJ. Collagen density promotes mammary tumor initiation and progression. BMC Med 2008 Apr;6(1):11.

4. Kalluri R, Zeisberg M. Fibroblasts in cancer. Nat Rev Cancer 2006 May;6(5):392-401.

5. Kim SH, Turnbull J, Guimond S. Extracellular matrix and cell signalling: the dynamic cooperation of integrin, proteoglycan and growth factor receptor. J Endocrinol 2011 May;209(2): 139-151.

6. Pietras K, Ostman A. Hallmarks of cancer: interactions with the tumor stroma. Exp Cell Res 2010 May;316(8): 1324-1331.

7. Bancroft, JD.; Gamble, M. Theory and practice of histological techniques. Philadelphia (PA): Elsevier Health Sciences; 2008.

8. Campagnola PJ, Dong C-Y. Second harmonic generation microscopy: principles and applications to disease diagnosis. Laser Photon Rev 2011 Jan;5(1):13-26.

9. Rich A, Crick FHC. The structure of collagen. Nature 1955 Nov;176:915-916.

10. Murray, RK.; Granner, DK.; Mayes, PA.; Rodwell, VW. The text book of harpers biochemistry. Los Altos (CA): Appleton and Lange, Norwalk, Connecticut; 1991.

11. Vasudevan, DM.; Sreekumari, S. Text book of biochemistry. New Delhi: Jay Pee Brothers; 2016.

12. Miller N. Ten Cate's oral histology. Br Dent J 2012 Aug; 213(4):194.
13. Fang M, Yuan J, Peng C, Li Y. Collagen as a double-edged sword in tumor progression. Tumour Biol 2014 Apr;35(4): 2871-2882.

14. Page-McCaw A, Ewald AJ, Werb Z. Matrix metalloproteinases and the regulation of tissue remodelling. Nat Rev Mol Cell Biol 2007 Mar;8(3):221-233.

15. Huijbers IJ, Iravani M, Popov S, Robertson D, Al-Sarraj S, Jones $\mathrm{C}$, Isacke CM. A role for fibrillar collagen deposition and the collagen internalization receptor endo180 in glioma invasion. PLoS One 2010 Mar;5(3):e9808.

16. Paszek MJ, Zahir N, Johnson KR, Lakins JN, Rozenberg GI, Gefen A, Reinhart-King CA, Margulies SS, Dembo M, Boettiger D, et al. Tensional homeostasis and the malignant phenotype. Cancer Cell 2005 Sep;8(3):241-254.

17. Kumari K, Ghosh S, Patil S, Augustine D, Venkatesiah SS, Roa RS. Expression of type III collagen correlation with poor prognosis in OSCC. J Invest Clin Dent 2016; 00:1-7.

18. Xiao Q, Ge G. Lysyl oxidase, extracellular matrix remodeling and cancer metastasis. Cancer Microenviron 2012 Dec;5(3):261-273.

19. Ebihara T, Venkatesan N, Tanaka R, Ludwig MS. Changes in extracellular matrix and tissue viscoelasticity in bleomycininduced lung fibrosis. Temporal aspects. Am J Respir Crit Care Med 2000 Oct;162(4 Pt 1):1569-1576.

20. Robins SP, Milne G, Duncan A, Davies C, Butt R, Greiling D, James IT. Increased skin collagen extractability and proportions of collagen type III are not normalized after 6 months healing of human excisional wounds. J Invest Dermatol 2003 Aug;121(2):267-272.

21. Arnold SA, Rivera LB, Miller AF, Carbon JG, Dineen SP, Xie Y, Castrillon DH, Sage EH, Puolakkainen P, Bradshaw AD, et al. Lack of host SPARC enhances vascular function and tumor spread in an orthotopic murine model of pancreatic carcinoma. Dis Model Mech 2010 Jan-Feb;3(1-2):57-72.

22. Shieh AC. Biomechanical forces shape the tumor microenvironment. Ann Biomed Eng 2011 May;39(5):1379-1389.

23. Karsdal MA, Nielsen MJ, Sand JM, Henriksen K, Genovese F, Bay-Jensen AC, Smith V, Adamkewicz JI, Christiansen C, Leeming DJ. Extracellular matrix remodeling: the common denominator in connective tissue diseases possibilities for evaluation and current understanding of the matrix as more than a passive architecture, but a key player in tissue failure. Assay Drug Dev Technol 2013 Mar;11(2):70-92.

24. Provenzano PP,Eliceiri KW,CampbellJM,Inman DR, WhiteJG, Keely PJ. Collagen reorganization at the tumor-stromal interface facilitates local invasion. BMC Med 2006 Dec; 4(1):38.

25. Conklin MW, EickhoffJC, Riching KM, PehlkeCA, Eliceiri KW, Provenzano PP, Friedl A, Keely PJ. Aligned collagen is a prognostic signature for survival in human breast carcinoma. Am J Pathol 2011 Mar;178(3):1221-1232.

26. Bredfeldt JS, Liu Y, Conklin MW, Keely PJ, Mackie TR, Eliceiri KW. Automated quantification of aligned collagen for human breast carcinoma prognosis. J Pathol Inform 2014 Aug;5(1):28.

27. Brabrand A, Kariuki II, Engstrøm MJ, Haugen OA, Dyrnes LA, Åsvold BO, Lilledahl MB, Bofin AM. Alterations in collagen fibre patterns in breast cancer. A premise for tumour invasiveness? APMIS 2015 Jan;123(1):1-8.

28. Campagnola P. Second harmonic generation imaging microscopy: applications to diseases diagnostics. Anal Chem 2011 May;83(9):3224-3231. 
29. Strupler M, Pena AM, Hernest M, Tharaux PL, Martin JL, Beaurepaire E, Schanne-Klein MC. Second harmonic imaging and scoring of collagen in fibrotic tissues. Opt Express 2007 Apr;15(7):4054-4065.

30. Drifka CR, Loeffler AG,Mathewson K, Mehta G, Keikhosravi A, Liu Y, LemancikS, Ricke WA, Weber SM, Kao WJ, Eliceiri KW. Comparison of picrosirius red staining with second harmonic generation imaging for the quantification of clinically relevant collagen fiber features in histopathology samples. J Histochem Cytochem 2016 Sep;64(9):519-529.

31. Matteini P, Ratto F, Rossi F, Cicchi R, Stringari C, Kapsokalyvas D, Pavone FS, Pini R. Photothermally-induced disordered patterns of corneal collagen revealed by SHG imaging. Opt Express 2009 Mar;17(6):4868-4678.
32. Thrasivoulou C, Virich G, Krenacs T, Korom I, Becker DL. Optical delineation of human malignant melanoma using second harmonic imaging of collagen. Biomed Opt Express 2011 May;2(5):1282-1295.

33. Watson JM, Rice PF, Marion SL, Brewer MA, Davis JR, Rodriguez JJ, Utzinger U, Hoyer PB, Barton JK. Analysis of second-harmonic-generation microscopy in a mouse model of ovarian carcinoma. J Biomed Opt 2012 Jul;17(7): 0760021-9.

34. Pirhonen J, Arola J, Sädevirta S, Luukkonen P, Karppinen SM, Pihlajaniemi T, Isomäki A, Hukkanen M, Yki-Järvinen $H$, Ikonen E. Continuous grading of early fibrosis in NAFLD using label-free imaging: a proof-of-concept study. PloS One 2016 Jan;11(1):e0147804. 